\title{
Spin-polarized currents in exchange spring systems
}

\author{
Matteo Franchin, ${ }^{1,2}, *$ Giuliano Bordignon, ${ }^{1,2}$ Thomas Fischbacher, ${ }^{2}$ Guido \\ Meier, ${ }^{3}$ Jürgen Zimmermann, ${ }^{2}$ Peter de Groot, ${ }^{1}$ and Hans Fangohr ${ }^{2}$ \\ ${ }^{1}$ School of Physics and Astronomy, University of Southampton, SO17 1BJ Southampton, United Kingdom \\ ${ }^{2}$ School of Engineering Sciences, University of Southampton, SO17 1BJ Southampton, United Kingdom \\ ${ }^{3}$ Institut für Angewandte Physik und Zentrum für Mikrostrukturforschung, \\ Universität Hamburg, Jungiusstrasse 11, 20355 Hamburg, Germany
}

(Dated: January 23, 2008)

\begin{abstract}
We present a computational study of the magnetization dynamics of a tri-layer exchange spring system in the form of a cylindrical nano-pillar, in the presence of an electric current. A three dimensional micromagnetic model is used, where the interaction between the current and the local magnetization is taken into account following a recent model by Zhang and Li. We obtain a stationary rotation of the magnetization of the system around its axis, accompanied by a compression of the artificial domain wall in the direction of the electron flow.
\end{abstract}

\section{INTRODUCTION}

The effects of spin-polarized currents on the magnetization of a ferromagnet have received considerable interest in recent times [1-3], after being proposed and studied in earlier works [4]. Spin-polarized currents may be used to generate microwave oscillations in the magnetization of a ferromagnet [5] or to switch the magnetization of a memory element [6]. The limit to these applications is currently found in the magnitude of the current density required to obtain significant effects, which is of the order of $10^{10}-10^{12} \mathrm{~A} / \mathrm{m}^{2}$. Consequently, there is a strong interest in finding novel devices where spin-torque effects are enhanced and require lower current density. The recent discovery of significant GMR in exchange spring multilayers [7], suggests that spin-transfer-torque may play a role in these systems. A further reason, which makes exchange spring systems attractive, is the possibility of creating "artificial" domain walls. Their length and shape - whose importance has been recently emphasized [8] - can be controlled, first during the engineering phase, then by applying a suitable magnetic field [7].

\section{THE SYSTEM}

Consider a system whose ground state energy is degenerate: it has infinitely many different equilibrium configurations, which all have the same minimal energy and form a continuous curve in the phase space. This system can be "dragged" through this curve, changing its configuration from one equilibrium state to another and this can be achieved "easily", because there is no energy barrier between them. In such a system, an electric current may find a very favorable situation to fully manifest its effects.

The idea is very simple, but can serve as a guideline to develop micromagnetic systems where spin-transfertorque effects are maximized. In this paper we discuss a possible example of such a system. We study a tri-

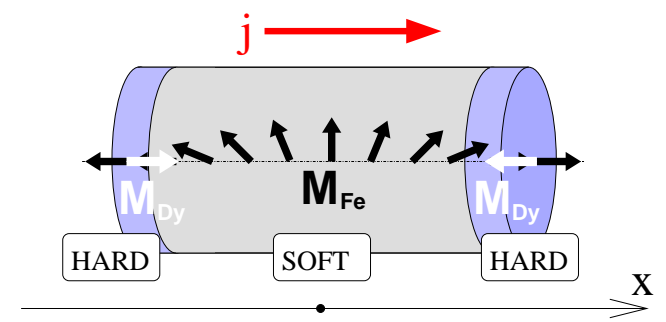

FIG. 1: A sketch of the nano-pillar which is studied in the paper (not to scale). Dysprosium moments (white arrows) pin the iron moments (black arrows) at the borders of the soft layer.

layer exchange spring system in the form of a cylindrical nano-pillar, where a central magnetically soft layer is sandwiched between two magnetically hard layers, as shown in Fig. 1. The system materials are chosen in the following way: $\mathrm{YFe}_{2}$ for the soft layer and $\mathrm{DyFe}_{2}$ for the two hard layers. This choice allows us to study the system with a model similar to the one used in our previous work [9]. Regarding the geometry, the diameter of the cylindrical nano-pillar is $10 \mathrm{~nm}$, while the thicknesses of the hard and soft layers are 5 and $40 \mathrm{~nm}$, respectively.

Yttrium has negligible magnetic moment and only two species of atoms contribute to the magnetization of the system: the first one, iron $(\mathrm{Fe})$, is present in all the three layers, the second one, dysprosium (Dy), is present only in the two hard layers. Neighboring iron moments are exchange coupled, throughout all the hard and soft layers and across the hard-soft interfaces. This coupling favors the alignment of the magnetization of iron throughout the entire nano-pillar. This alignment is however broken, because the magnetization of iron in the two hard layers is pinned along opposite directions, as shown in Fig. 1. The pinning of the iron moments is the result of the joint actions of two strong interactions: the cubic anisotropy of $\mathrm{DyFe}_{2}$, which pins the dysprosium moments along an easy axis direction, and the anti-ferromagnetic coupling Dy-Fe which transmits this pinning to the iron moments 
of the hard layers.

Among all the interactions which we take into account, the cubic anisotropy of $\mathrm{DyFe}_{2}$ is the only one which is not symmetric under rotations around the axis of the nanopillar. However in the case we are considering, where there is no applied field and the soft layer is relatively thick, the dysprosium moments keep their direction well aligned with the one they initially have and the degeneracy of the ground state is well preserved, as we will see from the results of the computer simulations. This means that configurations which differ by a rotation around the $x$ axis have almost the same energy. Then if the applied current wants to rotate the whole magnetization around the $x$ axis, nothing will oppose to its action.

\section{THE MODEL}

Since the density of iron atoms and their position in the lattice structure is the same for $\mathrm{DyFe}_{2}$ and $\mathrm{YFe}_{2}$ (they both crystallize in laves phase structures), we use a single magnetization vector $\mathbf{M}_{\mathrm{Fe}}$ to describe the magnetic configuration of iron in all the three layers. The configuration of dysprosium is modeled by another magnetization field $\mathbf{M}_{\mathrm{Dy}}$ which is defined over the hard layers only. The model is similar to the one-dimensional model used in [9], extended to three dimensions (the stray field is calculated using the hybrid FEM/BEM method $[10,11]$ ). We also consider the same temperature $(100 \mathrm{~K})$ and the same material parameters: the moment densities of iron (in both $\mathrm{DyFe}_{2}$ and $\left.\mathrm{YFe}_{2}\right)$ and dysprosium are $\left\|\mathbf{M}_{\mathrm{Fe}}\right\|=0.55 \times$ $10^{6} \mathrm{~A} / \mathrm{m}$ and $\left\|\mathbf{M}_{\text {Dy }}\right\|=1.73 \times 10^{6} \mathrm{~A} / \mathrm{m}$, respectively; the easy axes for the anisotropy are $\mathbf{u}_{1}=(0,1,1) / \sqrt{2}$, $\mathbf{u}_{2}=(0,-1,1) / \sqrt{2}$ and $\mathbf{u}_{3}=(1,0,0)$, and the coefficients are $K_{1}=33.9 \times 10^{6} \mathrm{~J} / \mathrm{m}^{3}, K_{2}=-16.2 \times 10^{6} \mathrm{~J} / \mathrm{m}^{3}$, $K_{3}=16.4 \times 10^{6} \mathrm{~J} / \mathrm{m}^{3}$. The effects of the electric current are modelled using the Zhang-Li correction to the Landau-Lifshitz-Gilbert equation [12]. We assume that only the iron moments interact with the spin of the conduction electrons: the magnetic electrons in the $4 \mathrm{f}$ orbitals of dysprosium are strongly localized at the ion core and their interaction with the conduction electrons should be negligible. In the simulation the damping parameter is chosen to be $\alpha=0.02$; the current density is assumed to be fully polarized $(P=1)$ and $\xi$, the ratio between the exchange relaxation time and the spin-flip relaxation time, is taken to be $\xi=0.01$. The Oersted field and the effects of Joule heating are ignored in the present study.

\section{RESULTS}

The simulations are performed by Nmag [13], a FEMbased (Finite Element Method) micromagnetic simulation package. The cylindrical nano-pillar is modelled by a three-dimensional unstructured mesh and first order FEM is used to discretize the space. In this case FEM

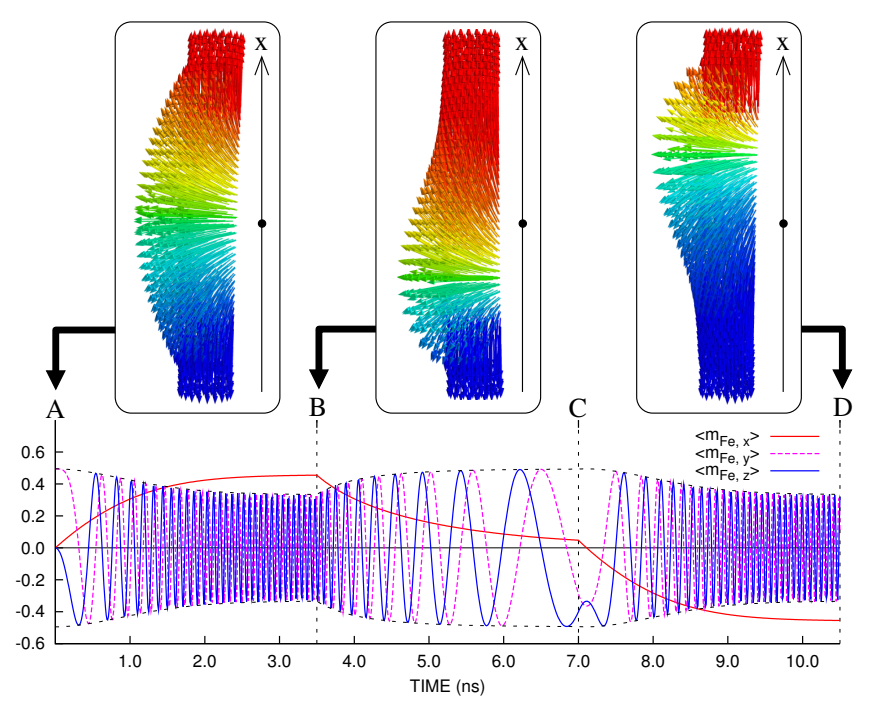

FIG. 2: The evolution in time of the three components of $\left\langle\mathbf{m}_{\mathrm{Fe}}\right\rangle=\left\langle\mathbf{M}_{\mathrm{Fe}} /\left\|\mathbf{M}_{\mathrm{Fe}}\right\|\right\rangle$, the normalized magnetization of iron averaged over all the nano-pillar. The three boxes above the graph show the configuration of $\mathbf{M}_{\mathrm{Fe}}$ at $t=0, t=3.5 \mathrm{~ns}$ and $t=10.5 \mathrm{~ns}$.

is preferable with respect to finite differences, because it allows a better representation of the cylindrical geometry. Finite differences would introduce artifacts in the discretization of the rounded surface of the nano-pillar.

The initial magnetizations $\mathbf{M}_{\mathrm{Fe}}$ and $\mathbf{M}_{\mathrm{Dy}}$ are obtained by letting the system relax to one of its degenerate equilibrium configurations. The system then evolves from this configuration $(t=0)$ up to $t=10.5 \mathrm{~ns}$. The dynamics of $\left\langle\mathbf{M}_{\mathrm{Fe}}\right\rangle$, the iron magnetization averaged over all the nano-pillar, is studied in Fig. 2. For simplicity we identify four points on the time axis: $\mathrm{A}$ at $0 \mathrm{~ns}$, B at $3.5 \mathrm{~ns}$, $\mathrm{C}$ at $7 \mathrm{~ns}$ and $\mathrm{D}$ at $10.5 \mathrm{~ns}$. The time axis is then subdivided into three regions $\mathrm{AB}, \mathrm{BC}$ and $\mathrm{CD}$. The applied current density $\mathbf{j}$ is uniform and constant in each of these three time intervals. In particular it is directed along the axis of the cylinder: $\mathbf{j}=j \mathbf{x}$, with $j=10^{11} \mathrm{~A} / \mathrm{m}^{2}$ in $\mathrm{AB}$, $j=0$ in $\mathrm{BC}$ and $j=-10^{11} \mathrm{~A} / \mathrm{m}^{2}$ in CD. We remind the reader that the applied field is always zero, throughout all the simulation.

The graph in Fig. 2 shows the behavior of the components of $\left\langle\mathbf{M}_{\mathrm{Fe}}\right\rangle$ : in region $\mathrm{AB}$ the current produces a precession of the whole magnetization of the system around the $x$ axis. This precession is accompanied by a movement - and consequent compression - of the artificial domain wall in the direction of the electron flow (negative $x$ direction), which reflects in an increase of the $x$ component of $\left\langle\mathbf{M}_{\mathrm{Fe}}\right\rangle$. Such an effect may be explained with a current-induced motion of the artificial domain wall. Current-induced motion is a well known effect for domain walls in nano-wires: it has been observed experimentally and has been proved analytically [14-16].

In the time interval $\mathrm{AB}$ the current pumps energy into 


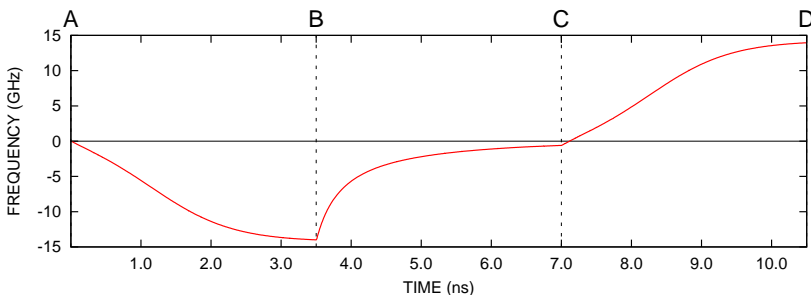

FIG. 3: The time dependence of the frequency $\omega$ for the precession of $\left\langle\mathrm{M}_{\mathrm{Fe}}\right\rangle$ around the $x$ axis. The sign of $\omega$ is related to the sense of rotation.

the system, which is stored in the compression of the domain-wall. In the time interval $\mathrm{BC}$ the current is switched off and this energy is gradually released: the domain-wall decompresses, restoring the configuration it had at time $t=0$. Finally, during the time interval CD the system behaves in a way which is symmetrical to the one observed in $\mathrm{AB}:\left\langle M_{\mathrm{Fe}, x}\right\rangle$ rotates in the opposite direction and the wall is compressed in the positive $x$ direction, leading to negative values for $\left\langle M_{\mathrm{Fe}, x}\right\rangle$.

Expressing $\left\langle\mathbf{M}_{\mathrm{Fe}}\right\rangle$ in spherical coordinates with $x$ chosen as the polar axis, we obtained the precession angle $\phi(t)$ of $\left\langle\mathbf{M}_{\mathrm{Fe}}\right\rangle$ around the $x$ axis as a function of time $t$. We computed the time derivative $\omega(t)=\phi^{\prime}(t)$ to obtain the precession frequency as a function of time. The result is shown in Fig. 3. The sign of $\omega(t)$ depends on the sense of rotation around the $x$ axis. This graph shows that the applied current $j= \pm 10^{11} \mathrm{~A} / \mathrm{m}^{2}$, produces a precession motion with frequency around $14 \mathrm{GHz}$, in the microwave frequency range. The frequency seems to be related to the compression of the domain wall: it increases rapidly when $\left\langle M_{\mathrm{Fe}, x}\right\rangle$ increases and stabilizes when also $\left\langle M_{\mathrm{Fe}, x}\right\rangle$ does.

The accuracy of the discretization of space has been verified by increasing the number of mesh elements (from 4129 to 19251), obtaining differences in the precession frequency at $3.5 \mathrm{~ns}$ lower than $1.2 \%$.

\section{DISCUSSION}

The model we presented does not take into account a number of effects which complicate the physics of real systems. The imperfections of the geometry and the impurities in the materials can break the cylindrical symmetry. The effect of such imperfections is difficult to predict.

The size of the sample was chosen to speed up the simulation. However we expect a similar precessional dynamics in nano-pillars with greater radii. Also the materials could have been chosen differently and the $\mathrm{DyFe}_{2}$ anisotropy could have been well approximated by an infinite pinning on the iron moments, resulting in a simplifi- cation of the model. However this approximation would have removed the only source of symmetry breaking, besides the irregularity of the unstructured mesh.

Other simulations should be carried out to understand how the precession frequency depends on the current density and what the role of the system geometry is.

To conclude we remark that a symmetry breaking could be introduced on purpose to obtain bistable systems, where the current may be used to switch the magnetization between two states.

This work has been funded by the Engineering and Physical Sciences Research Council (EPSRC) in the United Kingdom (GR/T09156, GR/S95824).

G. M. acknowledges financial support by the Deutsche Forschungsgemeinschaft via SFB 668 and GK 1286.

* franchin@soton.ac.uk

[1] J. C. Slonczewski, J. Magn. Magn. Mater. 159, L1 (1996).

[2] M. Tsoi, A. G. M. Jansen, J. Bass, W.-C. Chiang, M. Seck, V. Tsoi, and P. Wyder, Phys. Rev. Lett. 80, 4281 (1998).

[3] L. Berger, Phys. Rev. B 54, 9353 (1996).

[4] L. Berger, J. Appl. Phys. 49, 2156 (1978).

[5] J. A. Katine, F. J. Albert, R. A. Buhrman, E. B. Myers, and D. C. Ralph, Phys. Rev. Lett. 84, 3149 (2000).

[6] Y. Acremann, J. P. Strachan, V. Chembrolu, S. D. Andrews, T. Tyliszczak, J. A. Katine, M. J. Carey, B. M. Clemens, H. C. Siegmann, and J. Stöhr, Phys. Rev. Lett. 96, 217202 (2006).

[7] S. N. Gordeev, J.-M. L. Beaujour, G. J. Bowden, P. A. J. de Groot, B. D. Rainford, R. C. C. Ward, M. R. Wells, and A. G. M. Jansen, Phys. Rev. Lett. 87, 186808.1 (2001).

[8] M. Kläui, M. Laufenberg, L. Heyne, U. Rüdiger, C. A. F. Vaz, J. A. C. Bland, L. J. Heyderman, S. Cherifi, A. Locatelli, T. O. Mentes, et al., App. Phys. Lett. 88, 232507 (2006).

[9] M. Franchin, J. Zimmermann, T. Fischbacher, G. Bordignon, P. de Groot, and H. Fangohr, IEEE Transactions on Magnetics 43, 2887 (2007).

[10] D. R. Fredkin and T. R. Koehler, IEEE Trans. Magn. 26, 415 (1990).

[11] D. A. Lindholm, IEEE Trans. Magn. 20, 2025 (1984).

[12] S. Zhang and Z. Li, Phys. Rev. Lett. 93, 127204 (2004).

[13] Nmag - a micromagnetic simulation environment (2007), http://www. soton.ac.uk/ f angohr/nsim/nmag.

[14] B. Krüger, D. Pfannkuche, M. Bolte, G. Meier, and U. Merkt, Phys. Rev. B 75, 054421 (2007).

[15] G. Meier, M. Bolte, R. Eiselt, B. Krüger, D.-H. Kim, and P. Fischer, Phys. Rev. Lett. 98, 187202 (2007).

[16] M. Kläui, P.-O. Jubert, R. Allenspach, A. Bischof, J. A. C. Bland, G. Faini, U. Rüdiger, C. A. F. Vaz, L. Villa, and C. Vouille, Phys. Rev. Lett. 95, 026601 (2005). 